\title{
Efecto de la cocaína sobre la inhibición por prepulso de la respuesta de sobresalto
}

\author{
M. Carmen Arenas, Cristina Caballero-Reinaldo, Concepción I. Navarro-Francés, Carmen Manzanedo
}

Introducción. La inhibición por prepulso (IPP) de la respuesta de sobresalto es una medida de sincronización sensitivomotora basada en la respuesta del reflejo de sobresalto. Un déficit en la IPP se ha observado en pacientes psiquiátricos, especialmente con esquizofrenia, así como en sujetos vulnerables a desarrollarla. Asimismo, los consumidores de cocaína presentan un alto índice de patologías psiquiátricas como la esquizofrenia.

Objetivo. Conocer las alteraciones que el consumo de cocaína puede producir en la IPP.

Desarrollo. Se realiza una revisión exhaustiva de los estudios, tanto clínicos como con modelos animales, que hayan evaluado la IPP tras el consumo o la administración de cocaína. Se sugieren bases neurales y mecanismos de acción subyacentes para explicar los resultados.

Conclusiones. La cocaína altera la IPP a través de su acción sobre el sistema dopaminérgico. La administración aguda de cocaína disminuye la IPP al aumentar la dopamina, mientras que con el consumo crónico, dependiendo del tiempo de abstinencia, la IPP puede restablecerse. Sin embargo, los efectos de la cocaína sobre la IPP parecen depender de los niveles basales de la IPP que muestre el individuo. Así, dado que un déficit en la IPP se ha relacionado con una mayor vulnerabilidad a desarrollar patologías mentales como la esquizofrenia, los niveles de la IPP en los sujetos podrían considerarse como un biomarcador de vulnerabilidad psiquiátrica. Por ello, conocer mejor el efecto que drogas como la cocaína ejercen sobre la IPP puede ayudar a comprender el desarrollo de la patología dual.

Palabras clave. Cocaína. Dopamina. Inhibición por prepulso. Trastornos psiquiátricos.

\section{Introducción}

La inhibición por prepulso (IPP) se define como una medida de sincronización sensitivomotora basada en la respuesta del reflejo de sobresalto. Tiene lugar cuando un estímulo de alta intensidad es precedido de un estímulo menos intenso que provoca la atenuación de la respuesta del reflejo de sobresalto, de manera que, cuanto menor sea el nivel de la respuesta del reflejo de sobresalto mostrada, mayor será la IPP. Se trata de un proceso adaptativo para el sujeto, ya que lo aleja de una sobrecarga estimular. Este fenómeno sirve para regular la entrada sensorial, filtrando los estímulos irrelevantes o distractores y evitando así el desorden de información sensorial, lo que permite el procesamiento selectivo y eficiente de la información de carácter relevante [1-4].

Numerosos estudios han mostrado que la medida de la IPP puede ser útil en el ámbito de las enfermedades mentales, tanto en la investigación con seres humanos $[2,3,5]$ como utilizando modelos animales $[4,6]$. Se han observado déficits en la IPP en personas con distintos trastornos mentales en los que es significativa la presencia de anomalías en los mecanismos de inhibición sensoriales, cognitivos o mo- tores, y la esquizofrenia es el más característico [2, 7,8 . Dichos trastornos presentan también en su neurobiología un correlato anatómico y funcional común con la IPP $[3,4]$. En general, los resultados obtenidos desde hace años apuntan a que la IPP puede ser un marcador de vulnerabilidad para la esquizofrenia [4] y una herramienta a tener presente en la detección de síntomas y déficits cognitivos en otros muchos trastornos mentales [3-5,7,8].

Un área de investigación reciente y de gran interés es la relación entre la presencia de trastornos mentales y el consumo compulsivo de drogas. Los informes señalan que hasta el $70 \%$ de las personas diagnosticadas de un trastorno por abuso de sustancias presenta un trastorno psiquiátrico añadido, mientras que el $45-50 \%$ de los pacientes diagnosticados de un trastorno mental tienen antecedentes de un trastorno por abuso de sustancias $[9,10]$. Esta concurrencia de trastornos, denominada patología dual, implica una amplificación de la gravedad de cada trastorno con una peor evolución y además supone una mayor discapacidad funcional para el individuo. En poblaciones concretas, como personas en prisión o en la indigencia, enfermos, mujeres o personas mayores, la presencia de patología dual
Unidad de Investigación Psicobiología de las Drogodependencias. Departamento de Psicobiología. Facultad de Psicología. Universitat de València. Valencia, España.

Correspondencia:

Dra. Carmen Manzanedo. Departamento de Psicobiología. Facultad de Psicología. Universitat de València. Avda. Blasco Ibáñez, 21. E-46010 Valencia.

E-mail: carmen.manzanedo@uv.es

Financiación:

Proyecto I+D+i del Ministerio de Economía y Competitividad (MINECO) PSI2015-69649-R.

Aceptado tras revisión externa: 09.10.17.

Cómo citar este artículo: Arenas MC, Caballero-Reinaldo C, Navarro-Francés $\mathrm{Cl}$, Manzanedo C. Efecto de la cocaína sobre la inhibición por prepulso de la respuesta de sobresalto. Rev Neurol 2017; 65: 507-19.

(c) 2017 Revista de Neurología 
es aún mayor que en el resto de la población, y discurre con más morbilidad y mortalidad [11]. Por todo ello, la presencia de una patología dual constituye un grave problema de salud pública, principalmente en España, donde la población con patología dual es del 25-70\% según estudios [10].

En el caso concreto de los consumidores de cocaína, los estudios informan de la presencia de patología dual en el 50-75\%, es decir, estos sujetos, en su mayoría jóvenes, cumplen criterios diagnósticos de otro trastorno mental junto con el uso compulsivo de cocaína $[9,12,13]$. Hasta el momento se desconoce la dirección de esta relación, es decir, si el consumo de drogas precipita el desarrollo de un trastorno mental o si la vulnerabilidad al trastorno psiquiátrico desencadena el consumo. Sin embargo, sí parece que un contacto temprano con las drogas incrementa la probabilidad de desarrollar patología dual $[12,13]$.

Es muy conocida la acción de la cocaína sobre el sistema dopaminérgico [14] y las alteraciones neuronales que provoca su consumo [15]. Por otro lado, se sabe que este sistema de neurotransmisión es crucial en la regulación de la IPP $[3,4,6]$. Por ello, estamos interesados en estudiar cómo el consumo de esta droga puede provocar alteraciones en la IPP. Teniendo en cuenta que esta medida se considera un marcador de vulnerabilidad a patologías psiquiátricas, conocer el efecto de la cocaína sobre la IPP nos proporcionaría información sobre el papel que esta droga puede tener en el desarrollo de ciertos trastornos mentales. Así, el objetivo del presente trabajo es revisar los estudios que determinan cómo un consumo de cocaína, ya sea agudo o crónico, puede producir alteraciones en la IPP. Para ello realizamos una búsqueda exhaustiva de los estudios tanto clínicos como con modelos animales que hayan evaluado el efecto de la administración o el consumo de cocaína sobre el paradigma de la IPP.

\section{IPP del reflejo de sobresalto}

\section{Concepto}

La IPP se fundamenta en el reflejo de sobresalto, el cual es una respuesta espontánea, incondicionada y rápida, emitida en unos pocos milisegundos, ante una estimulación inesperada y de elevada intensidad, provocada por cualquier modalidad sensorial. Se caracteriza por la contracción coordinada de músculos esqueléticos junto con la aceleración del ritmo cardíaco. Es una respuesta que se impone a la conducta consciente que el individuo está realizan- do en ese momento. Sin embargo, si un estímulo de baja intensidad precede a la presentación del estímulo más intenso, el reflejo de sobresalto se atenúa. Es decir, aunque el reflejo de sobresalto es una reacción automática, su expresión se puede modular por la presencia previa de estímulos de menor intensidad. Este fenómeno es el que se conoce como IPP. Por lo tanto, la IPP es la reducción del reflejo de sobresalto debido a la presentación de un estímulo sensorial débil (llamado prepulso) que precede a la presentación del estímulo sensorial más intenso, generador del sobresalto (conocido como pulso). Así, el prepulso, que no causa el reflejo de sobresalto, interviene como barrera inhibitoria disminuyendo la respuesta al estímulo sensorial o pulso [1-4,8,16,17].

Este fenómeno se considera una medida operacional simple de procesamiento de la información sensorial y de sincronización sensitivomotora, caracterizada por su capacidad de filtrar la información relevante para el sujeto evitando una sobrecarga de estímulos $[3,8]$. La IPP es un proceso automático, que no se vincula al aprendizaje y que mantiene cierta estabilidad en el tiempo [18]. En general, se utilizan estímulos acústicos para generar la IPP, aunque puede provocarse con estímulos de cualquier modalidad sensorial (visual, cutánea, etc.). Además, se ha observado que se produce en un gran número de especies, lo que permite su uso tanto en la investigación básica como en la clínica [5]. En los seres humanos se mide el componente ocular del reflejo de sobresalto mediante el registro electromiográfico del músculo orbicular del ojo $[1,8,19]$. En roedores, se evalúa la respuesta motora esquelética de todo el cuerpo ante un estímulo auditivo [20]. Ambas evaluaciones tienen propiedades similares y reflejan la actividad de diferentes circuitos neuronales [21,22]. Neuroanatómicamente, el reflejo de sobresalto se genera en estructuras de la formación reticular del troncoencéfalo encargadas de integrar señales sensoriales [1-4,16,17]. Sin embargo, la IPP, al tratarse de un mecanismo inhibitorio de control/regulador, implica estructuras corticales y subcorticales $[3,8,16,17]$. Por consiguiente, esta medida puede utilizarse para evaluar la función de estructuras complejas [1-4].

La IPP se fundamenta en mecanismos principalmente automáticos preatencionales, por lo que puede observarse con intervalos entre los estímulos cortos (<60 ms), pero alcanza su máxima amplitud con intervalos entre los estímulos entre 60-120 ms. Cuando se utiliza un intervalo mayor (120-240 ms), es posible modular la IPP instruyendo a los sujetos en la atención hacia los estímulos. Así, con intervalos entre los estímulos mayores, el sujeto puede centrar 
su atención en el prepulso o en el pulso, para focalizarlo o ignorarlo, alterando con ello la IPP. Por lo tanto, como la IPP es un proceso automático, depende del diseño experimental empleado y también de las instrucciones dadas, de ahí la importancia de la unificación de la metodología utilizada $[5,18]$.

\section{Historia}

El término 'inhibición por prepulso' fue acuñado por Ison y Hammond en 1971. No obstante, existen antecedentes históricos relacionados con el término anteriores a esta fecha. En 1863, Sechenov describió por primera vez el reflejo de sobresalto, resultado de sus estudios sobre la neurofisiología de la inhibición del sistema nervioso central. Ya en el siglo xx, Peak demostró en 1939 la modulación del reflejo inhibitorio en el sistema auditivo. Pero fue en 1965 cuando Hoffman y Searle observaron que la respuesta del reflejo de sobresalto disminuía al ser precedida por un sonido; además, señalaron que esto se producía cuando el sonido se presentaba entre 20 y $500 \mathrm{~ms}$ antes del inicio del estímulo de sobresalto [23]. Más tarde, en 1978, el equipo de Braff demostró por primera vez los déficits en la IPP de los pacientes esquizofrénicos, lo que provocó un gran número de estudios que han revelado la relación entre este paradigma y la esquizofrenia [8].

Las primeras teorías explicativas de la IPP las encontramos en el modelo de procesamiento secuencial de la información que describía el cognitivismo clásico [24]. Pero fue a finales de los años setenta del siglo pasado, con el desarrollo de los modelos conexionistas, cuando se intentó explicar las alteraciones de la IPP observadas en los esquizofrénicos. La esquizofrenia se pasó a entender como un 'síndrome de desconexión' que afectaba a conexiones entre determinadas regiones corticales y subcorticales a través del tálamo [25], desconexiones que podrían ser responsables de los déficits observados en la IPP de los esquizofrénicos $[7,8]$.

Los estudios que utilizan el paradigma de la IPP, sobre todo en cuanto a la investigación traslacional se refiere, han aumentado desde mediados de los setenta, debido a dos factores complementarios entre sí. Por una parte, esta medida neurobiológica ha demostrado su utilidad para profundizar en los principios de la modulación del reflejo como mecanismo de filtración sensitivomotora; y, por otra, su déficit o alteración se han asociado con determinadas patologías mentales $[3,7,8]$. En estos últimos años se ha comenzado a utilizar esta prueba en el área de las drogodependencias. Se ha observado una alteración de la IPP en consumidores de diferentes
Figura. Esquema que representa las estructuras que intervienen en la producción de la inhibición por prepulso y sus conexiones. Flechas rojas: efecto de inhibición; flechas verdes: dirección de la información; flechas negras: circuito específico de integración de la información; flechas segmentadas: efecto de modulación.

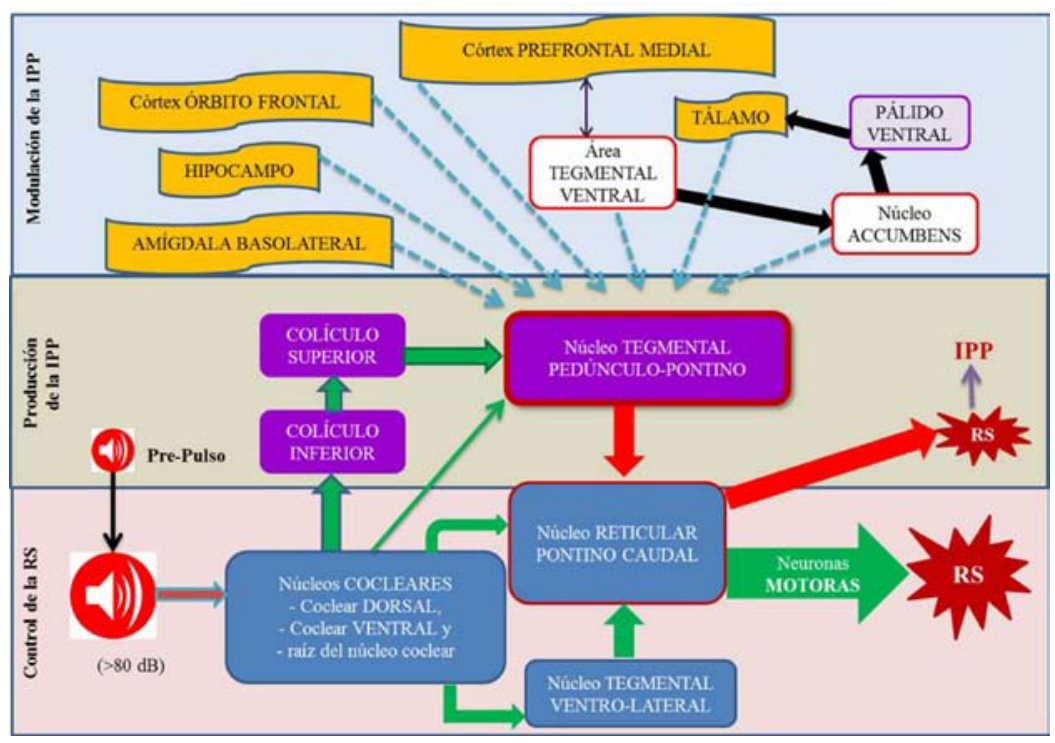

drogas, aunque son muy pocos los estudios realizados hasta el momento [3,5].

\section{Neurobiología de la IPP}

En el fenómeno de la IPP se distinguen tres partes diferenciadas: las estructuras que controlan la respuesta de sobresalto, las que desencadenan la IPP y las que modulan la IPP (Figura). Las estructuras que intervienen presentan conexiones neuronales con una actuación secuencial y paralela.

El circuito se inicia con la entrada de un input acústico en los núcleos cocleares (núcleo coclear dorsal, raíz del núcleo coclear y núcleo coclear ventral), que forman parte de la vía auditiva primaria. Cuando un estímulo acústico excede un determinado nivel de decibelios (> $80 \mathrm{~dB}$ ), la información pasa al núcleo tegmental ventrolateral y al núcleo pontinocaudal de la formación reticular. Este último es uno de los núcleos más basales del troncoencéfalo y tiene varias proyecciones que van directamente a las neuronas motoras, hecho que le confiere una notable implicación en el circuito primario de la respuesta del reflejo de sobresalto. En último lugar, las neuronas motoras activan la respuesta motora, lo cual completa el circuito de la respuesta del reflejo de sobresalto $[1,5]$. 
El input excitatorio que desencadena la IPP ante el estímulo auditivo se origina en el circuito auditivo del colículo inferior en el mesencéfalo, lugar adonde llega la información auditiva de los núcleos cocleares. El colículo inferior, a su vez, activa el colículo superior, cuyas proyecciones se dirigen hacia el núcleo tegmental pedunculopontino que inhibe al núcleo pontinocaudal de la formación reticular. La inhibición de este núcleo provoca una regulación a la baja de la respuesta de sobresalto, lo que permite medir el efecto de la IPP. Las estructuras que intervienen tienen diferente implicación. En cuanto a los colículos inferior y superior; el primero parece ser importante para la supresión de la respuesta del reflejo de sobresalto provocada por un estímulo auditivo, mientras que el colículo superior se considera crucial en la unión de la información proveniente de diferentes modalidades sensoriales (visual y táctil), aunque ambos colículos intervienen en la atenuación del efecto de la IPP, como se ha demostrado mediante estudios de lesiones [23]. El núcleo tegmental pedunculopontino también interviene en la mediación de la IPP, ya que su lesión parcial o total provoca una disrupción de la respuesta de la IPP y su estimulación produce una IPP [23]. En este circuito también interviene el núcleo tegmental laterodorsal, la pars reticulata de la sustancia negra [23] y la región dorsolateral de la sustancia gris periacueductal [17].

La modulación de la IPP se realiza mediante estructuras límbicas y corticales que presentan conexiones recíprocas. Entre las estructuras implicadas en esta modulación se incluyen el área tegmental ventral, la sustancia negra y el tálamo, adonde llegan aferencias provenientes de los núcleos tegmental pedunculopontino y tegmental laterodorsal. Las eferencias y conexiones del área tegmental ventral, la sustancia negra y el tálamo implican a su vez a otras áreas corticolímbicas, entre ellas el núcleo accumbens, el pálido ventral, la amígdala basolateral, el sistema septohipocámpico, y la corteza prefrontal medial y orbitofrontal. Ambas redes, tanto la que desencadena como la que modula, estarían en continua interacción para determinar la eficacia de la IPP, probablemente protegiendo mediante el prepulso o facilitando el procesamiento de sobresalto [17]. En líneas generales, este circuito límbico corticoestriadopalidopontino sería el que principalmente modula la IPP [3], y hay que señalar que esta activación límbica correlaciona en la IPP de ratas y de humanos [22].

Se ha sugerido que una mala comunicación neural entre las estructuras límbicas y corticales en el circuito corticoestriadopalidopontino puede ser la base de los déficits en la IPP. El núcleo accumbens se considera una estructura primordial en la regulación y alteración de la IPP, junto con las subregiones corticales y límbicas adyacentes. La manipulación del hipocampo o la amígdala basolateral, así como las disfunciones en la corteza prefrontal medial y corteza orbitofrontal también producen cambios en la IPP [3]. Pero, además, la modulación de la IPP depende de la interconexión entre las estructuras de todo el circuito corticoestriadopalidopontino [3]. Así, la implicación del hipocampo en la disrupción de la IPP es a través de la vía del núcleo accumbens, mientras que las conexiones eferentes del hipocampo a la corteza prefrontal medial también serían las responsables de algunas disrupciones de la IPP, como informan estudios anatómicos y funcionales [5]. Pero, además, la conexión alterada entre estructuras corticales y subcorticales puede provocar déficits en la modulación de la IPP a largo plazo. La lesión del núcleo paraventricular del tálamo deteriora la IPP de los animales, ya que se interrumpe la conexión con la corteza prefrontal medial, corteza encargada de regular la actividad dopaminérgica en el área tegmental ventral, lo que provoca que desaparezca el control que a través del glutamato ejerce la corteza sobre el sistema dopaminérgico mesolímbico [26]. En suma, diferentes áreas corticales y subcorticales están implicadas en la regulación de la IPP y, a través de diferentes vías, la modulación de estas estructuras puede causar una disrupción o reducción de la IPP. El circuito del reflejo de sobresalto se desinhibe y la entrada sensitivomotora se descompensa o desestabiliza. En tales casos se produciría una fuerte respuesta de sobresalto, aunque se presente el prepulso $[1,3,5]$.

En cuanto a la neuroquímica se refiere, la dopamina es el neurotransmisor de referencia tanto en la producción como en la modulación de la IPP, y las principales alteraciones en la IPP se relacionan con anomalías en este sistema de neurotransmisión $[3,4,6]$. Se considera que el sujeto con alteración de la función dopaminérgica es incapaz de filtrar la información irrelevante del entorno debido a que las funciones inhibitorias reguladas por este neurotransmisor están afectadas $[27,28]$. Se plantea que la respuesta del reflejo de sobresalto en estos sujetos permanecería alta, incluso con la presentación previa del prepulso, y, en consecuencia, la IPP sería menor. El nivel de dopamina en el núcleo accumbens es importante, ya que un aumento de dopamina disminuye la IPP, como se observa tras la administración de agonistas dopaminérgicos [29], y también lo es el nivel de dopamina prefrontal. La corteza prefrontal medial modula la señalización dopaminérgica del área tegmental ventral, por lo que 
variaciones en la interrelación de estas estructuras provocarían alteración de la dopamina en el núcleo accumbens, y se observarían déficits en la IPP [26]. Esta variabilidad, que puede existir en la producción y modulación de la IPP, se traduciría en diferencias en la función ejecutiva y control cognitivo entre individuos con alto o bajo nivel de IPP, como así se ha descrito; la formación de estrategias es más efectiva y el tiempo de ejecución es menor en los individuos con una alta IPP [30,31]. Sin embargo, el sistema dopaminérgico no es el único implicado. La desregulación de la IPP se ha relacionado con otros sistemas de neurotransmisión, entre los que se encuentran el serotoninérgico [32], el gabérgico [33], el glutamatérgico [4] y el colinérgico [34].

\section{IPP en los trastornos mentales}

Las patologías psiquiátricas que presentan una alteración dopaminérgica primordial comparten también un déficit en la IPP. Las variaciones en el nivel de dopamina y en la función dopaminérgica, como en la sensibilidad de los receptores, se ha comprobado que correlacionan con alteración cognitiva. En la clínica esto se traduce en dificultades en el procesamiento de la información como consecuencia de una inhibición sensorial inadecuada, provocando que los sujetos sean incapaces de centrarse en los aspectos más relevantes del entorno $[1,3,8]$. La esquizofrenia ha sido el trastorno psiquiátrico más relacionado con un déficit en la IPP [2], pero esta correlación también se ha observado en otras patologías, como los trastornos neurocognitivos y del desarrollo neurológico, la depresión, la ansiedad y la hiperactividad, entre otros $[2,5,8]$. Incluso en patologías que presentan una marcada alteración genética, como la enfermedad de Huntington, el síndrome de deleción 22q11 y el síndrome X frágil, también se ha detectado un déficit en la IPP [7]. Además, se han observado niveles más bajos en la IPP en familiares de pacientes esquizofrénicos y con trastorno bipolar que no estaban afectados por dichos trastornos [2,5]. Con lo expuesto, se plantea que la IPP puede constituir un endofenotipo neurofisiológico para la esquizofrenia y un rasgo genéticamente determinado, como indican las diferencias observadas en el índice de IPP, tanto en los pacientes como en sus familiares $[7,8]$.

La IPP se considera uno de los índices neurofisiológicos más prometedores de la investigación traslacional en psiquiatría $[4,5]$. Su interés y el beneficio de su uso se han demostrado en el ámbito de la patología mental, ya que detecta las diferencias individuales, se emplea tanto en humanos como en roe- dores de forma similar, y los sustratos neurales que la regulan están también implicados en diferentes patologías. Aunque no debe utilizarse como herramienta de diagnóstico clínico, ya que un déficit en la IPP también se observa en familiares sanos de esquizofrénicos, este paradigma puede ayudarnos a entender los procesos que contribuyen a presentar un déficit en los mecanismos inhibitorios conductuales. Es importante destacar que la hipótesis de trabajo actual no es que los síntomas clínicos se produzcan por un déficit en la IPP, sino que los procesos genéticos y neurobiológicos de estos dos procesos (los síntomas clínicos y el déficit de la IPP) se solapan. Por ello, se considera particularmente útil como medida conductual, la cual, combinada con otras pruebas y en un contexto experimental, puede describir alteraciones y vías desreguladas que se observan en condiciones clínicas $[5,8]$.

La relación de la IPP con la dopamina plantea su uso también en el ámbito de la adicción, y, aunque hasta el momento los estudios son escasos, existen evidencias que apuntan a su utilidad en los trastornos por uso de sustancias. Está demostrado que el consumo de drogas altera el sistema dopaminérgico mesolímbico [35], que también es el sistema responsable de la IPP $[3,6]$, y es de esperar, por ello, diferencias en la producción de la IPP según el consumo de drogas. Kumari et al [36] evalúan el cambio de la IPP antes y después de la administración de una dosis baja de anfetamina (activación del sistema) y observan que la IPP no varía en los sujetos que no fuman, mientras que disminuye en los fumadores. El estudio indica que el fenómeno de la IPP es sensible a la manipulación dopaminérgica, dependiendo de la experiencia previa con las drogas, y señala, en este caso, que el subgrupo de fumadores presenta sensibilización dopaminérgica, de tal modo que con el mismo estímulo se produce un mayor aumento en la liberación de dopamina. Se ha sugerido incluso que podría existir un componente genético heredable relacionado con el consumo de drogas, ya que se ha observado una IPP menor en los hijos de padres alcohólicos [37]. Así, la neuroplasticidad provocada por el consumo crónico de drogas se verá reflejada en alteraciones de la IPP.

\section{Cocaína}

La cocaína es la droga estimulante ilegal más consumida en Europa. El Observatorio Europeo de las Drogas y las Toxicomanías, en el informe realizado en 2016 , estima que el $1,9 \%$ de los adultos jóvenes de 15 a 34 años $(2,4$ millones de personas de este 
grupo de edad) consumió cocaína el año anterior. Concretamente, en España, alrededor de un 2,8\% de los adolescentes de 14-18 años consumió cocaína en el año 2014, y el 3,5\% admitió haber consumido esta droga alguna vez en la vida [38]. Los consumidores de esta sustancia constituyen el tercer grupo más numeroso que recurre a tratamientos ambulatorios, y son característicos en ellos el policonsumo y el trastorno mental. La población que consume cocaína como droga principal suele notificar también consumo de cannabis y alcohol como drogas secundarias, y muchos consumidores de opioides como droga principal notifican también un consumo secundario de cocaína [38].

La comorbilidad psiquiátrica es frecuente en el abuso de sustancias en general $[9,12]$, y entre los consumidores de cocaína en particular [13]. Como consecuencia, el pronóstico de la evolución de la adicción empeora [10], junto con todas las implicaciones clínicas y sociales que de ello se derivan [11]. Entre las psicopatologías más comunes encontradas en los consumidores de cocaína se han descrito la psicosis cocaínica, los trastornos de ansiedad, del estado de ánimo, del control de impulsos, de la personalidad (límite, antisocial, histriónico, narcisista, pasivo-agresivo y paranoide son los más frecuentes), por déficit atencional residual, estados disociativos, alteraciones cognitivas y disfunciones sexuales [13]. Por ello, cada vez más la investigación considera el estudio de la patología dual que presentan los individuos en tratamiento.

\section{Mecanismo de acción}

El principal mecanismo de acción de la cocaína reside en las vías dopaminérgicas mesolímbica y mesocortical. La cocaína bloquea la bomba de recaptación, es decir, el transportador de la dopamina, y, en consecuencia, se incrementa tanto la cantidad de dopamina como el tiempo de exposición de la dopamina en el núcleo accumbens y en el resto de estructuras que reciben las proyecciones del área tegmental ventral y la sustancia negra, entre las que resaltan la corteza prefrontal medial, los núcleos caudado y putamen, el hipocampo y la amígdala [35]. En estas estructuras, el aumento de dopamina activa los receptores pre y postsinápticos dopaminérgicos durante mucho tiempo, con lo que ya desde los primeros consumos se altera la señalización dopaminérgica y se van produciendo cambios secuenciales, progresivos y a largo plazo, dependiendo también del consumo posterior [35,39].

Con la administración crónica se ha observado que disminuye la síntesis de dopamina, y con ello su nivel endógeno, liberación y recambio. Disminuye también la disponibilidad de los transportadores y de los receptores D2/D3 [14], y de manera gradual, se va alterando la distribución e interrelación entre los receptores D1/D2, los cuales presentan hipersensibilidad postsináptica, y aumenta el número de receptores D3 [35,39]. A través de la activación de los receptores D1, la cocaína induce cambios moleculares activando la vía de segundos mensajeros, como el monofosfato de adenosina cíclico (AMPc). La vía del AMPc estimula la producción de proteínas intracelulares impulsando la expresión de genes de acción temprana, que en regiones como los ganglios basales y el núcleo accumbens son responsables del desarrollo de rigidez en la respuesta conductual, formación de hábitos y conducta compulsiva que caracteriza al trastorno por abuso de sustancias [35, 39]. Por otro lado, las modificaciones en la expresión y señalización de estos receptores se relacionan con la aparición de trastorno mental en individuos que no han consumido cocaína [40].

No obstante, aunque las acciones de la cocaína sobre el sistema dopaminérgico se consideran prioritarias, también incrementa los niveles de noradrenalina y, en menor medida, de serotonina, pues bloquea los transportadores de estos dos neurotransmisores, los cuales son muy similares al transportador de la dopamina, impidiendo su recaptación presináptica. Además, el sistema glutamatérgico es de especial importancia en la neuroplasticidad que induce la cocaína. La administración de cocaína produce hiperexcitabilidad en las neuronas de dopamina a través de los receptores NMDA y AMPA situados en los terminales dopaminérgicos del área tegmental ventral [35]. Este primer cambio en el disparo neuronal del área tegmental ventral altera la comunicación con el núcleo accumbens, y es el inicio de las siguientes transformaciones sinápticas a nivel de las estructuras con las que conecta el área tegmental ventral y el núcleo accumbens, como la corteza prefrontal medial y la amígdala, entre otras [41]. La regulación dopaminérgica del área tegmental ventral al núcleo accumbens y la modulación de la corteza prefrontal medial al núcleo accumbens son críticas en el desarrollo del trastorno por abuso de sustancias, y también parecen serlo en la esquizofrenia [27].

Así, con el consumo crónico se van produciendo cambios más permanentes, de manera secuencial, en la estructura y el funcionamiento celular del cerebro, que envuelven la corteza y estructuras subcorticales. Se ha observado en los cocainómanos un menor volumen de sustancia gris en la corteza, la amígdala y el estriado [39], y también un patrón anormal de actividad y metabolismo cerebral 
Tabla I. Estudios que evalúan el efecto de la cocaína sobre la inhibición por prepulso en humanos.

\begin{tabular}{|c|c|c|c|c|}
\hline & Fármaco & Sexo & Protocolo de consumo & Inhibición por prepulso \\
\hline \multirow{2}{*}{ Efferen et al [43] } & \multirow{2}{*}{$\begin{array}{c}\text { Cocaína } \\
\text { (50 dólares día/ } \\
200 \text { dólares binge) }\end{array}$} & \multirow{2}{*}{ Hombres } & Seis meses/binge fin de semana & Aumenta \\
\hline & & & Consumo negativo el día de la prueba & Aumenta \\
\hline \multirow{3}{*}{ Preller et al [44] } & \multirow{3}{*}{$\begin{array}{c}\text { Cocaína } \\
\text { (consumo mínimo } \\
1 \mathrm{~g} / \mathrm{mes} \text { ) }\end{array}$} & $\begin{array}{l}\text { Hombres } \\
\text { y mujeres }\end{array}$ & $\begin{array}{l}\text { Consumo de cocaína reciente } \\
\text { (muestra de orina positiva) }\end{array}$ & $\begin{array}{c}\text { Normal frente a controles } \\
\text { Disminuye frente a negativos en la orina }\end{array}$ \\
\hline & & $\begin{array}{l}\text { Hombres } \\
\text { y mujeres }\end{array}$ & $\begin{array}{l}\text { Consumo de cocaína no reciente } \\
\text { (muestra de orina negativa) }\end{array}$ & $\begin{array}{l}\text { Aumenta frente a controles } \\
\text { Aumenta frente a positivos en la orina }\end{array}$ \\
\hline & & $\begin{array}{l}\text { Hombres } \\
\text { y mujeres }\end{array}$ & $\begin{array}{l}\text { Consumidores recreacionales } \\
\text { y dependientes de la cocaína }\end{array}$ & Igual \\
\hline
\end{tabular}

en áreas corticales (prefrontal, parietal y temporal) y en estructuras subcorticales como el estriado respecto a las personas que no han consumido cocaína [15]. Además, es frecuente la presencia de déficits cognitivos (menor puntuación en pruebas de atención y memoria) y en funciones ejecutivas (menor rendimiento respecto a procesos de flexibilidad e inhibición) en dependientes de la cocaína [42]. La alteración cognitiva y ejecutiva se relacionaría con disfunciones del lóbulo frontal, y las alteraciones neuropsiquiátricas, que también presentan los adictos a la cocaína, se relacionarían con el deterioro de la función dopaminérgica. Las alteraciones en el sistema nigroestriado se relacionan con la aparición de sintomatología motora y extrapiramidal, como se observa en la enfermedad de Parkinson o el síndrome de Gilles de la Tourette, y en el sistema mesolímbico se relacionan con la aparición de delirio, psicosis y alteración de la conciencia, como se observa en las demencias o en la esquizofrenia [39].

\section{Relación entre la IPP y el consumo de cocaína}

El objetivo principal de este trabajo es establecer una relación entre el consumo de cocaína y las alteraciones en la IPP. Así, los trabajos revisados informan que el consumo de cocaína provoca variaciones en la IPP, pero no son muchos y los resultados no siempre coinciden. Sólo dos trabajos han evaluado la alteración de la IPP en relación con el consumo de cocaína en humanos (Tabla I). El primero resalta el aumento de determinados parámetros de la IPP en consumidores habituales de cocaína respecto a sujetos que no consumen [43] y el segundo pone de relieve que la IPP oscila dependiendo de la historia de consumo que realiza el sujeto [44]. Los estudios con modelos animales permiten controlar la historia de consumo de los sujetos, aunque este tipo de estudios son escasos: cinco con ratas (Tabla II) y dos con ratones (Tabla III). Estos trabajos muestran que la alteración de la IPP por efecto de la cocaína depende de la actividad dopaminérgica en la vía mesolímbica y está afectada por variables como el tipo de administración de la droga, ya sea aguda o crónica, y el tiempo de abstinencia, es decir, por las neuroadaptaciones inducidas por la cocaína.

\section{Efectos de la cocaína sobre la IPP}

La IPP se ha observado incrementada tanto en consumidores recreacionales como en dependientes de la cocaína con respecto al grupo control [44]. Sin embargo, cuando se controla su consumo reciente a través de un análisis de orina, la IPP es menor en los consumidores que dan positivo en la orina que en los consumidores que dan negativo. Esto último parece indicar que la IPP del sujeto disminuye bajo los efectos agudos de la cocaína, y se observa un incremento 'rebote' en la IPP durante la abstinencia. De hecho, los consumidores de cocaína abstinentes mostraron una mayor IPP en comparación con los controles [43]. Estos cambios en la IPP son consistentes con las neuroadaptaciones provocadas por el consumo continuado de cocaína, en concreto con un bajo nivel de dopamina en el estriado que tiende a recuperarse con la abstinencia $[14,35,45]$. Esta regulación se ha observado también en pacientes dependientes del alcohol. Todos presentaban una menor IPP que los controles, pero la IPP fue mejorando (es decir, aumentando) tras el tratamiento de desintoxicación, aunque sin alcanzar los niveles de inhibición de los controles sin dependencia [19]. 
Tabla II. Estudios que evalúan el efecto de la cocaína sobre la inhibición por prepulso en ratas.

\begin{tabular}{|c|c|c|c|c|}
\hline & Fármaco & Sexo & Protocolo de administración & Inhibición por prepulso \\
\hline & & & Aguda IP & Disminuye (20 y $30 \mathrm{mg} / \mathrm{kg}$ ) \\
\hline \multirow[t]{3}{*}{ Martínez et al [48] } & $\begin{array}{l}\text { Cocaína ( } 10,20 \text { y } 30 \text { mg/kg) } \\
\text { Cocaína (implantación 120, }\end{array}$ & Hembras & Crónica 0-5 días SC & Disminuye el día 3 \\
\hline & & & Abstinencia de 10 días & Restaura frente a controles \\
\hline & & & Aguda IP & Disminuye \\
\hline \multirow[t]{2}{*}{ Byrnes y Hammer [47] } & Cocaína (30mg/kg) & Machos & Crónica 7 días IP & Aumenta \\
\hline & & & Abstinencia de 7 días & Restaura frente a controles \\
\hline \multirow[t]{2}{*}{ Zhang et al [29] } & Cocaína (0,68 a 5,44 mg/kg) & Machos & Aguda IP & Sin cambios (dosis bajas) \\
\hline & & & Crónica IP: 15 días/8 semanas & \\
\hline \multirow[t]{2}{*}{ Adams et al [60] } & Cocaína (30mg/kg) & Machos & Abstinencia de 3 días & Sin cambios \\
\hline & & & Abstinencia de 14 días & Sin cambios \\
\hline Broderick y Rosenbaum [46] & Cocaína $(10,20$ y 40 mg/kg) & Machos y hembras & Aguda IP & Disminuye $(20 \mathrm{mg} / \mathrm{kg})$ \\
\hline
\end{tabular}

Los estudios realizados con animales corroboran esta conclusión. La administración aguda de altas dosis de cocaína disminuye la IPP en ratas [46-48] y ratones $[49,50]$. También provoca la disminución de la IPP, tanto en seres humanos [21,51, $52]$ como en ratas $[29,52,53]$ y ratones $[54,55]$, la administración aguda de otro psicoestimulante similar a la cocaína, como la anfetamina. De manera similar, otras drogas que consiguen aumentar la liberación de dopamina a través de mecanismos indirectos, como el antagonismo de receptores NMDA o la activación de receptores $5-\mathrm{HT}_{2 \mathrm{~A}}$, también provocan la disminución de la IPP. Así, la fenciclidina y la ketamina $[4,54]$, y las drogas alucinógenas con perfil serotoninérgico (LSD, psilocibina, dimetiltriptamina y MDMA) disminuyen la IPP en humanos, ratas y ratones [32], ya que de manera indirecta también incrementan la función dopaminérgica en el núcleo accumbens [27,56,57]. Es bien sabido que esta alteración de la vía dopaminérgica mesolímbica provoca, en mayor o menor medida, una potente adicción [58] y posibles episodios psicóticos, observados tanto en humanos como en modelos animales [59].

Pero los estudios con animales han permitido ampliar y aclarar más estos resultados. Zhang et al [29] demostraron que la IPP observada depende del nivel existente de dopamina en el núcleo accumbens. Comprobaron que la IPP sólo disminuye cuando se provoca un potente aumento de dopamina en dicha región utilizando para ello una dosis alta de anfetamina, pero que no varía cuando el aumento de dopamina es menor al utilizar una dosis mínima de cocaína. De acuerdo con ellos, Broderick y Rosenbaum [46] observaron que la dosis de $20 \mathrm{mg} / \mathrm{kg}$ de cocaína produce una mayor disminución de la IPP que la de $10 \mathrm{mg} / \mathrm{kg}$, de manera similar tanto en ratas machos como en hembras. Pero, teniendo en cuenta que la administración continuada de cocaína provoca una regulación a la baja de la función dopaminérgica [35], tras un consumo continuado de la droga debería observarse una regulación al alza de la IPP, de manera similar que con la abstinencia, cuando se recupera la actividad dopaminérgica normal. Tres de los estudios realizados con ratas han comprobado esta hipótesis [47,48,60]. Así, Martínez et al [48] observaron que la IPP de ratas con un implante subcutáneo de cocaína durante cinco días era menor que los controles al tercer día del implante, y volvía a ser similar a los 10 días después de retirar los implantes. De manera similar, Adams et al [60] evaluaron el efecto de la administración crónica de cocaína sobre la IPP con dos protocolos de administración, uno corto de dos se- 
Tabla III. Estudios que evalúan el efecto de la cocaína sobre la inhibición por prepulso en ratones.

\begin{tabular}{|c|c|c|c|c|c|}
\hline & Fármaco & Sexo & Modificación genética & Protocolo de administración & Inhibición por prepulso \\
\hline \multirow{2}{*}{$\begin{array}{l}\text { Yamashita } \\
\text { et al [49] }\end{array}$} & \multirow{2}{*}{$\begin{array}{c}\text { Cocaína } \\
\text { (30 y } 60 \text { mg/kg) }\end{array}$} & \multirow{2}{*}{ Machos } & Modificado DAT & Aguda intraperitoneal & Normaliza (30 y 60 mg/kg) \\
\hline & & & Control DAT & Aguda intraperitoneal & Disminuye con 60 mg/kg \\
\hline \multirow{2}{*}{$\begin{array}{l}\text { Doherty } \\
\text { et al [50] }\end{array}$} & \multirow{2}{*}{$\begin{array}{c}\text { Cocaína } \\
(40 \mathrm{mg} / \mathrm{kg})\end{array}$} & \multirow{2}{*}{$\begin{array}{l}\text { Machos y } \\
\text { hembras }\end{array}$} & Modificado receptor D1, D2, D3 & Aguda intraperitoneal & Sin efectos/Disminuye/Disminuye \\
\hline & & & Control receptor D1, D2 y D3 & Aguda intraperitoneal & Disminuye/Disminuye/Disminuye \\
\hline
\end{tabular}

DAT: transportador de dopamina.

manas y otro largo de ocho semanas, y no observaron diferencias en la IPP de los animales con los controles ni a los tres ni a los 14 días de finalizar cada tratamiento. Byrnes y Hammer [47] realizaron una administración crónica de cocaína para desarrollar sensibilización conductual. Como era de esperar, el primer día de tratamiento (administración aguda de cocaína) disminuyó la IPP de las ratas, pero, tras siete días de tratamiento, se observó un aumento de la IPP. Tras un intervalo de siete días después de finalizar la administración crónica, pusieron a prueba la presencia de sensibilización conductual. Administraron para ello una nueva dosis aguda de cocaína y comprobaron que la IPP volvía a disminuir. Por tanto, en resumen, parece ser que la liberación de dopamina inducida por la administración aguda de cocaína disminuye la IPP, mientras que la regulación de la actividad dopaminérgica que se va produciendo durante la abstinencia a la cocaína produce un aumento progresivo de la IPP.

Podría plantearse que tras una abstinencia prolongada la IPP se normalizaría, como parecen indicar los resultados con animales $[47,48,60]$; sin embargo, el patrón de consumo de la cocaína en los seres humanos es intermitente desde su inicio, con períodos más o menos largos de abstinencia y múltiples recaídas. Las neuroadaptaciones que se van desarrollando en la abstinencia se manifiestan ante nuevos contactos con la cocaína. En ratas con experiencia de autoadministración y tras 30 días de abstinencia, la cocaína provoca una mayor liberación de dopamina en el núcleo accumbens [61], mientras que los estímulos ambientales provocan una menor liberación de dopamina en el núcleo accumbens [62]. En sujetos dependientes de la cocaína también aparece alterada la respuesta del circuito de recompensa cerebral (en particular, un recambio de dopamina reducido en el núcleo accumbens y el área tegmental ventral), así como en el resto de estructuras del circuito corticoestriadopalidopontino, en especial la conectividad entre ellas. Así, se observa una disminución en la conectividad funcional entre el tálamo y la corteza prefrontal medial [28], y entre la corteza prefrontal medial y el núcleo accumbens [41]. Estos cambios se expresarían con diferente oscilación de la IPP y, a largo plazo, se podrían relacionar con la presencia de un trastorno por abuso de sustancias o patología dual.

Aunque está bien asentado que el aumento de dopamina disminuye la IPP, mientras que una reducción de dopamina no tiene efectos o aumenta la IPP, esta correspondencia va más allá de una relación lineal entre el nivel de IPP y la concentración de dopamina. En sujetos sanos que presentan una baja respuesta de IPP, ésta se puede aumentar con la administración de clozapina (un antagonista de la dopamina del receptor D4), lo que no ocurre en sujetos con una mayor IPP basal [63]. Esta variación de la IPP dependiendo de la situación basal también se ha observado con la administración de memantina (un antagonista de los receptores NMDA glutamatérgicos). La memantina aumenta la IPP en individuos considerados como bajos en su IPP, mientras que no produce alteración o tiende a disminuirla en los que presentan una mayor IPP [4]. Por tanto, la IPP presente en cada individuo parece constituir un índice indicativo de la respuesta que se va a producir ante la manipulación farmacológica. Algo similar ocurre tras la administración de una dosis baja de alcohol, donde la variación producida en la IPP está determinada por la situación basal que presentaban los sujetos en el inicio de la prueba [64]. El alcohol disminuía la IPP en los sujetos que presentaban baja inhibición, pero la aumentaba en los de alta IPP. Los autores señalan que la línea basal de la IPP puede ser indicativa de la respuesta al alcohol porque, en su estudio, no observaron otras diferen- 
cias en cuanto a la respuesta al alcohol (por ejemplo, afecto negativo o positivo y efecto estimulante o sedativo) [64]. Resultados similares se han obtenido utilizando anfetamina, con reducción de la IPP sólo en las mujeres consideradas en situación basal como altas en la IPP [21].

Consistente con estos resultados, los estudios con modelos animales han mostrado también que la IPP observada tras la administración de una droga es dependiente de la línea basal en la IPP de los sujetos, la cual a su vez se relaciona con la función dopaminérgica basal que presenta el animal. La anfetamina disminuye la IPP en las ratas que tienen una mayor IPP de línea basal [21]. También, en las ratas que presentan diferente sensibilidad a la apomorfina (un agonista dopaminérgico), la administración de cocaína disminuye la IPP sólo en los animales categorizados por su alta sensibilidad [65]. De igual manera, la anfetamina disminuye la IPP con dosis muy bajas en los animales más sensibles a la apomorfina, mientras que se necesitan dosis mayores para obtener el mismo efecto en las ratas menos sensibles [66]. Además de diferencias en la respuesta a la cocaína y a la anfetamina, las ratas más sensibles a la apomorfina presentan diferencias en la respuesta dopaminérgica ante la novedad, como es el aumento de dopamina y de la enzima tiroxina hidroxilasa en el núcleo accumbens $[67,68]$. Utilizando ratas con preferencia al alcohol, se han obtenido resultados que apuntan en la misma dirección. Si bien la administración de una dosis alta de anfetamina disminuye la IPP en todas las ratas, la disminución de la IPP observada es significativamente mayor en los animales que muestran una preferencia por el alcohol [69]. Así pues, entendiendo que la alteración dopaminérgica provocada por el consumo de cocaína puede considerarse un factor primordial en la alteración de la IPP, la IPP presente en cada sujeto antes del consumo de cocaína podría determinar la respuesta a la droga y ser indicativa del estado de la función dopaminérgica, así como de las neuroadaptaciones que la cocaína pudiera desarrollar. Considerar esto puede ser primordial a la hora de entender la alteración dopaminérgica provocada por diferentes factores, como el consumo de drogas.

Para finalizar este apartado, hay que señalar que la línea basal de la IPP no debe considerarse per se indicativa de una alteración dopaminérgica. Sujetos considerados de alto riesgo para presentar psicosis muestran una reducción de la IPP respecto de los controles, pero el estudio no observó correlación significativa entre la concentración de dopamina estriatal y el nivel de IPP de cada grupo [70]. Es más bien la presencia de hipersensibilidad dopaminérgica y un aumento de dopamina en el mesolímbico [27], que se puede producir tanto por el uso de drogas (alucinógenos y psicoestimulantes) [32] como por la conjunción de estrés y factores genéticos [27], lo que se ha relacionado con la desconexión en las estructuras que regulan la IPP, como el tálamo y la corteza prefrontal, y con una menor IPP [71].

\section{Receptores de dopamina en la IPP}

El papel de los receptores dopaminérgicos en la IPP se ha estudiado mediante la estrategia de observar si el bloqueo de los receptores de dopamina reduce o altera la disminución de la IPP provocada con agonistas dopaminérgicos (anfetamina, apomorfina, cocaína o agonistas selectivos de los receptores dopamina), utilizando para ello animales con y sin modificación genética. La estrategia se apoya en la premisa de que los cambios en la sensibilidad dopaminérgica, el nivel de dopamina o el volumen de receptores de dopamina determinan la IPP.

Así, parece que es más efectiva la activación de los D2 respecto de los D1 para obtener la disminución de la IPP cuando se usan agonistas selectivos $[29,72]$. Sin embargo, cuando se utilizan antagonistas, es el bloqueo de los receptores D1 el que revierte la disminución en la IPP que provoca la apomorfina, mientras que no es efectivo el bloqueo de los receptores D2 [73]. Doherty et al [50] comprobaron que el bloqueo de los receptores D1 y D2/D3 impide que la cocaína disminuya la IPP. Utilizando ratones con modificación genética para los receptores D1, D2 y D3, estos autores observaron que la cocaína no afecta la IPP de los animales sin receptor D1, pero produce una mayor disminución de la IPP en los ratones sin receptor D2 y una disminución mucho mayor en los animales sin receptor D3 que en sus controles. Debemos tener en cuenta que, como era de esperar, la administración aguda de cocaína disminuyó la IPP en los ratones control de cada receptor. A la vista de los resultados, los autores consideran que se produce un sinergismo entre los receptores, y es necesaria la activación de los receptores D1 para potenciar el efecto de los receptores D2 en la disminución de la IPP, pero es difícil concretar la actuación de cada uno por separado. Para finalizar, hay que señalar que el trabajo realizado por Yamashita et al [49] con ratones sin el transportador de la dopamina muestra que la cocaína no provoca disminución de la IPP en ellos, y pone de relieve nuevamente que es el aumento de dopamina que induce la cocaína el responsable de la disminución de la IPP. 


\section{Conclusiones}

La patología dual asociada al consumo de cocaína es bien conocida entre los profesionales. Muchos de los trastornos psiquiátricos que presenta el consumidor de cocaína (trastornos de la personalidad y de ansiedad, depresión, etc.) comparten déficits en la IPP, principalmente en el sistema de inhibición sensorial, así como alteraciones en el sistema de neurotransmisión dopaminérgica. No obstante, el origen de esta patología dual asociada al consumo de cocaína está aún por determinar. Se desconoce todavía si es el trastorno mental el que desencadena un consumo de la droga (en muchas ocasiones para aplacar la sintomatología clínica de la patología) o, por el contrario, es el propio consumo de cocaína el que precipita la aparición de otra patología psiquiátrica. Al mismo tiempo, se considera que la IPP es un endofenotipo para la esquizofrenia, ya que un déficit en este paradigma se relaciona con vulnerabilidad a desarrollar este trastorno psiquiátrico. Por todo ello, entender las alteraciones que un consumo agudo o crónico de cocaína provocan en la IPP puede ayudarnos a conocer la relación entre el consumo de drogas y el desarrollo de patologías psiquiátricas.

Los efectos del consumo de cocaína sobre el fenómeno de la IPP son complejos, y tal vez por ello se han realizado tan pocos estudios en comparación con otros agonistas dopaminérgicos. En esta revisión hemos puesto de manifiesto que el consumo de cocaína altera la IPP, debido principalmente a los cambios que la droga provoca en el sistema dopaminérgico. En concreto, se ha observado que la administración aguda de cocaína, al aumentar la disponibilidad de la dopamina, disminuye la IPP; mientras que un consumo crónico de la droga no parece alterar la IPP, ya que los sujetos presentan valores similares a los de los controles. Sin embargo, estos resultados dependen mucho del tiempo de abstinencia y de los patrones de consumo, por lo que podríamos hablar de un restablecimiento de los niveles de la IPP por las neuroadaptaciones observadas en el sistema dopaminérgico tras el consumo continuado. Es bien conocido que, independientemente del patrón de consumo realizado, la cocaína produce alteraciones tanto en el sistema de recompensa cerebral como en el corticoestriadopalidopontino, circuito regulador de la IPP.

Además, en esta revisión se ha puesto de relieve que los cambios en la IPP de los sujetos expuestos a diferentes manipulaciones farmacológicas van a depender de la situación basal que presente el individuo en la IPP. Como el fenómeno de la IPP refleja el estado del procesamiento de la información pre- atencional en el individuo, y un déficit en la IPP se ha relacionado con una mayor vulnerabilidad a desarrollar patologías mentales como la esquizofrenia, los niveles de la IPP en los sujetos podrían considerarse como un biomarcador de vulnerabilidad psiquiátrica. Por ello, conocer mejor el efecto que las drogas ejercen sobre la IPP puede ayudar a comprender el desarrollo de la patología dual. Asimismo, consideramos que sería de gran interés realizar más estudios que evalúen la respuesta de los individuos a drogas como la cocaína teniendo en cuenta el nivel basal de la IPP de los individuos. De esta manera se podría plantear el papel que puede llegar a tener la IPP en el ámbito clínico de la patología dual y en la detección precoz de este tipo de trastornos.

\section{Bibliografía}

1. Valls-Solé J. Funciones y disfunciones de la reacción de sobresalto en el ser humano. Rev Neurol 2004; 39: 946-55.

2. García-Sánchez F, Martínez-Gras I, Rodríguez-Jiménez R, Rubio G. Inhibición prepulso del reflejo de la respuesta de sobresalto en los trastornos neuropsiquiátricos. Rev Neurol 2011; 53: 422-32.

3. Swerdlow NR, Braff DL, Geyer MA. Sensoriomotor gating of the startle reflex: what we said 25 years ago, what has happened since then, and what comes next. J Psychopharmacol 2016; 30: 1072-81.

4. Swerdlow NR, Light GA. Animal models of deficient sensorimotor gating in schizophrenia: are they still relevant? Curr Top Behav Neurosci 2016; 28: 305-25.

5. Kohl S, Heekeren K, Klosterkötten J, Kuhn J. Prepulse inhibition in psychiatric disorders -apart from schizophrenia. J Psychiatr Res 2013; 47: 445-52.

6. Vargas JP, Díaz E, Portavella M, López JC. Animal models of maladaptive traits: disorders in sensorimotor gating and attentional quantifiable responses as possible endophenotypes. Front Psychol 2016; 7: 206.

7. Braff D, Greenwood TA, Swerdlow NR, Light GA, Schork NJ; Consortium on the Genetics of Schizophrenia. Avances en la endotipificación de la esquizofrenia. World Psychiatry (Ed Esp) 2008; 7: 11-8

8. Braff DL. Prepulse inhibition of the startle reflex: a window on the brain in schizophrenia. Curr Top Behav Neurosci 2010; 4: 349-71.

9. Arias F, Szerman N, Vega P, Mesías B, Basurte I, Morant C, et al. Abuso o dependencia a la cocaína y otros trastornos psiquiátricos. Estudio Madrid sobre la prevalencia de la patología dual. Revista de Psiquiatría y Salud Mental 2013; 6: 121-8.

10. Roncero C, Szerman N, Terán A, Pino C, Vázquez JM, Velasco E, et al. Professionals' perception on the management of patients with dual disorders. Patient Prefer Adherence 2016; 10: $1855-68$.

11. Szerman N, Marín-Navarrete R, Fernández-Mondragón J, Roncero C. Patología dual en poblaciones especiales: una revisión narrativa. Revista Internacional de Investigación en Adicciones 2015; 1: 50-67.

12. Arias F, Szerman N, Vega P, Mesías B, Basurte I, Rentero D. Trastorno bipolar y trastorno por uso de sustancias. Estudio Madrid sobre prevalencia de patología dual. Adicciones 2017; 29: 186-94.

13. González-Llona I, Tumuluru S, González-Torres MA, Gaviria M Cocaína: una revisión de la adicción y el tratamiento. Revista de la Asociación Española de Neuropsiquatría 2015; 35: 555-71.

14. Ashok AH, Mizuno Y, Volkow ND, Howes OD. Association of stimulant use with dopaminergic alterations in users of 
cocaine, amphetamine, or methamphetamine: a systematic review and meta-analysis. JAMA Psychiatry 2017; 74: 511-9.

15. Verdejo-García A, Pérez-García M, Sánchez-Barrera M, Rodríguez-Fernández A, Gómez-Río M. Neuroimagen y drogodependencias: correlatos neuroanatómicos del consumo de cocaína, opiáceos, cannabis y éxtasis. Rev Neurol 2007; 44: 432-9.

16. Koch M, Schnitzler HU. The acoustic startle response in ratscircuits mediating evocation, inhibition and potentiation. Behav Brain Res 1997; 89: 35-49.

17. Rohleder C, Wiedermann D, Neumaier B, Drzezga A Timmermann L, Graf R, et al. The functional networks of prepulse inhibition: neuronal connectivity analysis based on FDG-PET in awake and unrestrained rats. Front Behav Neurosci 2016; $10: 148$.

18. Swerdlow NR, Bhakta SG, Rana BK, Kei J, Chou HH, Talledo JA Sensorimotor gating in healthy adults tested over a 15 year period. Biol Psychol 2017; 123: 177-86.

19. Marín-Mayor M, Jurado-Barba R, Martínez-Grass I, PonceAlfaro G, Rubio-Valladolid G. La respuesta de sobresalto y la inhibición prepulso en los trastornos por uso de alcohol. Implicaciones para la práctica clínica. Clínica y Salud 2014; 25: 147-55.

20. Valsamis B, Schmid S. Habituation and prepulse inhibition of acoustic startle in rodents. J Vis Exp 2011; 55: e3446.

21. Talledo JA, Sutherland Owens AN, Schortinghuis T, Swerdlow NR. Amphetamine effects on startle gating in normal women and female rats. Psychopharmacology (Berl) 2009; 204: 165-75.

22. Kumari V, Antonova E, Zachariah E, Galea A, Aasen I, Ettinger U, et al. Structural brain correlates of prepulse inhibition of the acoustic startle response in healthy humans. Neuroimage 2005; 26: 1052-8.

23. Fendt M, Li L, Yeomans JS. Brain stem circuits mediating prepulse inhibition of the startle reflex. Psychopharmacology (Berl) 2001; 156: 216-24.

24. Fierro M. El desarrollo conceptual de la ciencia cognitiva. Parte I. Revista Colombiana de Psiquiatría 2011; 40: 519-33.

25. Andreasen NC. A unitary model of schizophrenia: Bleuler's 'fragmented phrene' as schizencephaly. Arch Gen Psychiatry 1999; 56: 781-7.

26. Öz P, Kaya Yertutanol FD, Gözler T, Özçetin A, Uzbay IT. Lesions of the paraventricular thalamic nucleus attenuates prepulse inhibition of the acoustic startle reflex. Neurosci Lett 2017; 642: 31-6.

27. Howes OD, McCutcheon R, Owen MJ, Murray RM. The role of genes, stress, and dopamine in the development of schizophrenia. Biol Psychiatry 2017; 81: 9-20.

28. Zhang S, Hu S, Bednarski SR, Erdman E, Li CS. Error-related functional connectivity of the thalamus in cocaine dependence. Neuroimage Clin 2014; 4: 585-92.

29. Zhang J, Forkstam C, Engel JA, Svensson L. Role of dopamine in prepulse inhibition of acoustic startle. Psychopharmacol (Berl) 2000; 149: 181-8.

30. Bitsios P, Giakoumaki SG, Theou K, Frangou S. Increased prepulse inhibition of the acoustic startle response is associated with better strategy formation and execution times in healthy males. Neuropsychologia 2006; 44: 2494-9.

31. Giakoumaki SG, Bitsios P, Frangou S. The level of prepulse inhibition in healthy individuals may index cortical modulation of early information processing. Brain Res 2006; 1078: 168-70.

32. Halberstadt AL, Geyer MA. Effect of hallucinogens on unconditioned behavior. Curr Top Behav Neurosci 2017; Feb 22. [Epub ahead of print].

33. Fendt M. Enhancement of prepulse inhibition after blockade of GABA activity within the superior colliculus. Brain Res 1999; 833: 81-5.

34. Bosch D, Schmid S. Cholinergic mechanism underlying prepulse inhibition of the startle response in rats. Neuroscience 2008; 155: 326-35.

35. Volkow ND, Morales $M$. The brain on drugs: from reward to addiction. Cell 2015; 162: 712-25.

36. Kumari V, Mulligan OF, Cotter PA, Poon L, Toone BK,
Checkley SA, et al. Effects of single oral administrations of haloperidol and d-amphetamine on prepulse inhibition of the acoustic startle reflex in healthy male volunteers. Behav Pharmacol 1998; 9: 567-76.

37. Grillon C, Sinha R, Ameli R, O'Malley SS. Effects of alcohol on baseline startle and prepulse inhibition in young men at risk for alcoholism and/or anxiety disorders. J Stud Alcohol 2000; 61: 46-54.

38. Observatorio Europeo de las Drogas y las Toxicomanías. Encuesta sobre uso de drogas en enseñanzas secundarias en España (ESTUDES). Informe 2016: alcohol, tabaco y drogas ilegales en España. Madrid: Ministerio de Sanidad, Servicios Sociales e Igualdad; 2016.

39. Cadet JL, Bisagno V, Milroy CM. Neuropathology of substance use disorders. Acta Neuropathol 2014; 127: 91-107.

40. Ledonne A, Mercuri NB. Current concepts on the physiopathological relevance of dopaminergic receptors. Front Cell Neurosci 2017; 11: 27.

41. Wang X, Liu L, Adams W, Li S, Zhang Q, Li B, et al. Cocaine exposure alters dopaminergic modulation of prefrontoaccumbens transmission. Physiol Behav 2015; 145: 112-7.

42. Lorea I, Fernández-Montalvo J, Tirapu-Ustárroz J, Landa N, López-Goñi JJ. Rendimiento neuropsicológico en la adicción la cocaína: una revisión crítica. Rev Neurol 2010; 51: 412-26.

43. Efferen TR, Duncan EJ, Szilagyi S, Chakravorty S, Adams JU, Gonzenbach S, et al. Diminished acoustic startle in chronic cocaine users. Neuropsychopharmacology 2000; 22: 89-96.

44. Preller KH, Ingold N, Hulka LM, Vonmoos M, Jenni D, Baumgartner MR, et al. Increased sensorimotor gating in recreational and dependent cocaine users is modulated by craving and attention-deficit/hyperactivity disorder symptoms. Biol Psychiatry 2013; 73: 225-34.

45. Volkow ND, Koob GF, McLellan AT. Neurobiologic advances from the brain disease model of addiction. N Engl J Med 2016; 374: 363-71.

46. Broderick PA, Rosenbaum T. Sex-specific brain deficits in auditory processing in an animal model of cocaine-related schizophrenic disorders. Brain Sci 2013; 3: 504-20.

47. Byrnes JJ, Hammer RP. The disruptive effect of cocaine on prepulse inhibition is prevented by repeated administration in rats. Neuropsychopharmacology 2000; 22: 551-4.

48. Martínez ZA, Ellison GD, Geyer MA, Swerdlow NR. Effects of sustained cocaine exposure on sensorimotor gating of startle in rats. Psychopharmacol (Berl) 1999; 142: 253-60.

49. Yamashita M, Fukushima S, Shen HW, Hall FS, Uhl GR, Numachi Y, et al. Norepinephrine transporter blockade can normalize the prepulse inhibition deficits found in dopamine transporter knockout mice. Neuropsychopharmacology 2006; 31: 2132-9.

50. Doherty JM, Masten VL, Powell SB, Ralph RJ, Klamer D, Low MJ, et al. Contributions of dopamine D1, D2, and D3 receptor subtypes to the disruptive effects of cocaine on prepulse inhibition in mice. Neuropsychopharmacology 2008; 33: 2648-56.

51. Hutchison KE, Swift R. Effect of d-amphetamine on prepulse inhibition of the startle reflex in humans. Psychopharmacology 1999; 143: 394-400.

52. Swerdlow NR, Stephany N, Wasserman LC, Talledo J, Shoemaker J, Auerbach PP. Amphetamine effects on prepulse inhibition across-species: replication and parametric extension. Neuropsychopharmacology 2003; 28: 640-50.

53. Zhang J, Engel JA, Söderpalm B, Svensson L. Repeated administration of amphetamine induces sensitisation to its disruptive effect on prepulse inhibition in the rat. Psychopharmacology 1998; 135: 401-6.

54. Dulawa SC, Geyer MA. Psychopharmacology of prepulse inhibition in mice. Chin J Physiol 1996; 39: 139-46.

55. Ralph RJ, Varty GB, Kelly MA, Wang YM, Caron MG, Rubinstein M, et al. The dopamine D2, but not D3 or D4 receptor subtype is essential for the disruption of prepulse inhibition produced by amphetamine in mice. J Neurosci 1999; 19: 4627-33.

56. Yavas E, Young AM. N-Methyl-D-aspartate modulation of nucleus accumbens dopamine release by metabotropic 
glutamate receptors: fast cyclic voltammetry studies in rat brain slices in vitro. ACS Chem Neurosci 2017; 8: 320-8.

57. Borroto-Escuela DO, Romero-Fernández W, Narváez M, Oflijan J, Agnati LF, Fuxe K. Hallucinogenic 5-HT2AR agonists LSD and DOI enhance dopamine D2R protomer recognition and signaling of D2-5-HT2A heteroreceptor complexes. Biochem Biophys Res Commun 2014; 443: 278-84.

58. Dominici P, Kopec K, Manur R, Khalid A, Damiron K, Rowden A. Phencyclidine intoxication case series study. J Med Toxicol 2015; 11: 321-5

59. Ham S, Kim TK, Chung S, Im HI. Drug abuse and psychosis: new insights into drug-induced psychosis. Exp Neurobiol 2017; 26: 11-24.

60. Adams JU, Efferen TR, Duncan EJ, Rotrosen J. Prepulse inhibition of the acoustic startle response in cocaine-withdrawn rats. Pharmacol Biochem Behav 2001; 68: 753-9.

61. Cameron CM, Wightman RM, Carelli RM. One month of cocaine abstinence potentiates rapid dopamine signaling in the nucleus accumbens core. Neuropharmacology 2016; 111: 223-30.

62. Saddoris MP. Terminal dopamine release kinetics in the accumbens core and shell are distinctly altered after withdrawal from cocaine self-administration. eNeuro 2016; 3. pii: ENEURO. 0274-16.2016.

63. Vollenweider FX, Barro M, Csomor PA, Feldon J. Clozapine enhances prepulse inhibition in healthy humans with low but not with high prepulse inhibition levels. Biol Psychiatry 2006; 60: 597-603.

64. Hutchison KE, Rohsenow D, Monti P, Palfai T, Swift R. Prepulse inhibition of the startle reflex: preliminary study of the effects of a low dose of alcohol in humans. Alcohol Clin Exp Res 1997; 21: 1312-9.

65. Van der Elst MC, Ellenbroek BA, Cools AR. Cocaine strongly reduces prepulse inhibition in apomorphine-susceptible rats, but not in apomorphine-unsusceptible rats: regulation by dopamine D2 receptors. Behav Brain Res 2006; 175: 392-8.
66. Van der Elst MC, Wunderink YS, Ellenbroek BA, Cools AR. Differences in the cellular mechanism underlying the effects of amphetamine on prepulse inhibition in apomorphine-susceptible and apomorphine-unsusceptible rats. Psychopharmacology (Berl) 2007; 190: 93-102.

67. Van der Elst MC, Verheij MM, Roubos EW, Ellenbroek BA, Veening JG, Cools AR. A single exposure to novelty differentially affects the accumbal dopaminergic system of apomorphinesusceptible and apomorphine-unsusceptible rats. Life Sci 2005; 76: 1391-406.

68. Van der Elst MC, Roubos EW, Ellenbroek BA, Veening JG, Cools AR. Apomorphine-susceptible rats and apomorphineunsusceptible rats differ in the tyrosine hydroxylaseimmunoreactive network in the nucleus accumbens core and shell. Exp Brain Res 2005; 160: 418-23.

69. Bell RL, Rodd ZA, Hsu CC, Lumeng L, Murphy JM, McBride WJ. Amphetamine-modified acoustic startle responding and prepulse inhibition in adult and adolescent alcohol-preferring and -nonpreferring rats. Pharmacol Biochem Behav 2003; 75: 163-71.

70. De Koning MB, Bloemen OJ, Van Duin ED, Booij J, Abel KM, De Haan L, et al. Pre-pulse inhibition and striatal dopamine in subjects at an ultra-high risk for psychosis. J Psychopharmacol 2014; 28: 553-60.

71. Falkai P, Rossner MJ, Schulze TG, Hasan A, Brzózka MM, Malchow B, et al. Kraepelin revisited: schizophrenia from degeneration to failed regeneration. Mol Psychiatry 2015; 20: 671-6.

72. Geyer MA, Krebs-Thomson K, Braff DL, Swerdlow NR. Pharmacological studies of prepulse inhibition models of sensorimotor gating deficits in schizophrenia: a decade in review. Psychopharmacol (Berl) 2001; 156: 117-54.

73. Ralph-Williams RJ, Lehmann-Masten V, Geyer MA. Dopamine D1 rather than D2 receptor agonists disrupt prepulse inhibition of startle in mice. Neuropsychopharmacology 2003; 28: 108-18.

\section{Effects of cocaine on prepulse inhibition of the startle response}

Introduction. Prepulse inhibition (PPI) of the startle response is an index used to evaluate how the pre-attention system works. PPI is altered in patients with a mental disorder such as schizophrenia and in subjects who are vulnerable to it. Likewise, cocaine users also frequently exhibit psychiatric disorders as schizophrenia.

Aim. To know the alterations that cocaine produces on PPI.

Development. A comprehensive review is carried out, covering both clinical and preclinical studies with animal models that have evaluated the effects of cocaine exposure on the PPI paradigm. Underlying neural bases and mechanisms of action are suggested to explain these findings.

Conclusions. Cocaine alters PPI through its action on the dopaminergic system. Acute exposure of cocaine decreases PPI by increasing dopamine, while with chronic use, depending on withdrawal time, PPI can be restored. However, the effects of cocaine on PPI appear to depend on the baseline levels of PPI shown by the individual. Thus, since a deficit in PPI has been associated with a greater vulnerability to developing mental pathologies such as schizophrenia, PPI level in subjects could be considered as a biomarker of psychiatric vulnerability. Therefore, a better understanding of the effect of drugs such as cocaine on PPI may help to understand the development of dual pathology.

Key words. Cocaine. Dopamine. Prepulse inhibition. Psychiatric disorders. 